\title{
Rectification and Explanation of Understandings of Computer
}

\author{
Ji Meng \\ School of Occupation Technical and Further Education, Hubei University for Nationalities, China \\ virusking@163.com
}

Keywords: Computer; understanding; rectification; explanation

\begin{abstract}
This paper rectifies and explains misunderstandings of computer hardware, file management, software system and virus defense, etc. It helps users to know and use the computer deeply from the details.
\end{abstract}

\section{Introduction}

China has entered the information society, as the basis of intelligent tools of computer productivity has played a very important role to promote the progress and development of the modern society. It has long been accustomed to the use of computer work and rich leisure life, it has already become an important tool, and one must rely on people's daily life, no doubt of its foundation status. It also facilitates the extensive development of the "better use of the computer and give full play to its effectiveness" for the purpose of various basic computer educations.

China has entered the information age, time is not long contacts with most of the crowd of computer tools have not experienced a system, a step-by-step process, together with basic computer education until recent years began to spread from the primary and secondary schools, must most non-computer science graduate and older people, the computer is set up in the "edge to edge fumble while summing up the practical application, which will inevitably result in computer awareness and application of the one-sidedness and limitations. The presence of some misunderstanding, to see from a short period of time will not give specific computer applications larger adverse impact, but in a longer period of time within some misunderstanding will ultimately affect and hinder the efficient computer tools to use. Therefore, the article will correct the mistake and eliminating computer awareness level term readers can continuously improve and able to promote the application of some common misconceptions.

\section{The Board Independent of the Computer Must be better than the Computer Integrated Boards}

Going back in time 10 years, the above theory is basically correct. Computer video signal and audio signal processing tasks undertaken by different parts of the hardware, in order to improve your computer's processing power, these processing unit hardware and software vendors to a single card in the form of independent, accompanied by professional enhancement processor for those graphics or sound computer user optional use of special processing requirements to meet the diversity of computer applications, which in a great degree, to provide a more flexible choice. This also left for the long-term normal use of the computer hidden - because the independent board with the communication between the computer's motherboard is dependent on the cheat card part of the motherboard slot between shrapnel tightly coupled, both in physical on contact can not be any disconnection occurs. A gold finger number of the contact surface is generally more than one hundred in the computer used for a long time, the air oxidation corrosion of a metal surface, the same time the motherboard slot of shrapnel will gradually lose flexibility, this will lead to poor contact between the two, and the computer hardware does not work. More than the existence of the problem, so that the loading independent board computers often use one to two years after 
intermittent, occasional emergence of various types of contact failure. Therefore, from the point of view of overall healthy functioning computer board independent of the computer may not like too integrated computer. As computer hardware technology matures, nowadays most of integrated board computer equipment also have a very strong performance, ordinary users to purchase a higher degree of integration of the computer equipment is more cost-effective, economic and save; course, for those who special application industry users, for example, music enthusiasts and graphic designers, is recommended according to the specific needs of the optional hardware devices with independent boards.

\section{The "Computer File" and "Computer Software" Distinction}

Any data are only stored as files on the computer, "document" constitutes the most basic computer storage unit (here refers to the formal can be felt from human).Computer software people manipulate a collection of class instruction using computer tools, the same store state must deposit in the form of files. A complete application software system contains a number of documents, after the software is activated, the normal operation of its function, which refers to the organization and coordination of these files between a complex call, this call by the prior defined somehow control rules, control rules when the software installation had been written to the operating system registry database. Once the software is running, it is responsible for the interaction between the registries. Although the software is stored in the form of files, but it also can modify the operation file. From the formal point of view, the software is just a collection of multiple files with a particular purpose.

\section{The Shortcut to the File and the File Itself Distinction}

Largely shortcuts and files in the Windows operating system, the file showed a certain similarity: mouse double-click the file and shortcut icon will cause the file to open; between the two icons are not too obvious difference, the only shortcut icon in the lower left corner of a small arrow with the small arrow (through some sort of system settings can also be removed, many people are likely to ignore this detail differences). The above similar point exists, often misunderstand beginners in understanding both the concept of the nature of them mixed up a class. Essentially, the shortcut file is generated by file basis, which directly point to the file, similar to a beacon. When the shortcut to execute a file "open" operation, the operation will automatically transfer to the entity files, so both run effect is consistent. Their differences are mainly reflected in the difference of the capacity, because shortcuts just play a simple point to the role and its size is generally not more than $2 \mathrm{~K}$; respective properties, the shortcut contain the entity files and information, and the file would only involve their own separate information.

\section{The File Name Modified Why cannot open the File}

In the Windows operating system, the full name of the file is composed of two parts, namely, "before the file name + extension, with a dot between the two parts as a continuation character. Before the file name is the conventional sense of the computer users think that the file name extension indicates that the file type. Windows operating system in the management of files, often through the extension of the file to identify the file type, and then call the corresponding already in the registry file handler or software related. For example, an extension of the text file called "TXT", the system default TXT file handler "Notepad" program, when the user double-clicks a TXT file, the background will automatically start the "Notepad" program to open the file computer users only with the help of the program in order to achieve the purpose of the operation on the file. Seen a complete file name must contain the correct extension portion, or the system will not be able 
to support the latter part of the operation of the user. The default file extension part of the Windows operating system is not hidden; any file name changes are likely to result in the extension part of the file changes. Renamed the operation is performed, the full name of the file in an editable state, beginners often forget the need to keep the extension part of the file unchanged, once rashly enter a new file name will cause the loss of extension section, naturally file it cannot be opened. In fact, to avoid this situation is very simple, only needs "a check on it in the" Folder Options "will" Hide extensions for known file types extensions.

\section{The More Complete the Operating System Patches Are, the Better}

The development of the operating system is a very complex and large project. The software vendor is prepared in the beginning in the design of the system, cannot be one hundred percent of the estimated various problems that may arise later use environment, so the operating system market and run a long time after its own all the problems will be gradually exposed out. In this case, the software vendors usually an occasional release of a series of targeted patch fixes to improve, in order to improve and strengthen the reliability and stability of the system. The patches involved a wide range of both system securities; The new version comes with a software upgrade. From a software system vendor perspective, the coverage of the patch is naturally the more complete the better, but for computer users in terms of the actual application environment, the choice of installation of the patch did not need to be too perfect. For example, is not networking using the computers do not have to consider the installation of network security patches. More because the number of programs installed on your computer, the corresponding direct consequence is the cause of the computer running efficiency decreased significantly. The overall effect is to consider applications from the computer; It is not necessary to install all the patches.

\section{Why the Computer is Used Longer, the Response Speed is slower}

The normal operation of the various types of software applications installed on top of the operating system, depends on the deployment and command within the system registry database. Therefore, the running speed of the various types of application software, in addition to the preparation of impact of the hardware platform, and the efficiency of the registry database read has a direct relationship. Software running will generate a certain number of temporary files, the location information of these files with the file generated automatically added to the registry database, but not with the software shutdown automatically clear. This caused two issues: First, a long time after the software repeatedly run will result in the temporary files generated during total amount is greatly increased, and will undoubtedly form many fragmented files on the hard disk, which will significantly reduce the reading efficiency of the computer system on the hard disk data; Secondly, no valid temporary file location information in the registry database increased, will continue to increase the capacity of the database file, reducing the reading efficiency of the data library. The above two points, will slow down the speed of response of the entire computer system. Therefore, the computer each used for a period of time, it is necessary to carry out a "disk defrag and clean up the registry operation [2], which helps to shorten the response time of the system, and improve work efficiency.

\section{After the Antivirus Software are Installed, the Computer must not be Infected}

Many people simply think that as long as the anti-virus software installed, you can sit back and relax, no longer need to fear of viruses, does not know that this understanding is extremely wrong. The spread of computer viruses has long past uncast to the full range of communication 
development, route of transmission of both With network vulnerabilities automatic background download With the removable media to spread a variety of ways, but also more subtle. The current phase of the anti-virus software protection technology to judge the suspicious file's behavior still not is completely intelligent, more often still need the participation of user interaction in order to carry out subsequent operations - to give the user prompt action for suspicious files, the file selected by the user whether to continue running. So, even if your computer is equipped with anti-virus software, also entirely possible user despise unfortunately being infected in the vast majority of antivirus conscious groups of people, the virus can easily fool them achieve the purpose of dissemination and parasitic. Therefore, after the installation of anti-virus software, in addition to the need to ensure the upgrade latest virus library data outside every day, which also requires the user to actively enrich protection knowledge, accumulated protection experience, try to reject the execution of unknown files so that they can at maximum The extent to achieve a better protective effect.

\section{Conclusion}

Awareness of Corrections and eliminating work through some of these contribute to a deeper understanding of computer tools to more effectively carry out a variety of applications for the specific use of the process better lay a good foundation help users from the details. Of course, due to space limitations, it may still exist a lot of misunderstanding has not been mentioned and readers feel, which requires users to constantly sum up the application process and learn, continue to correct misconceptions, so as to achieve the purpose of efficient use of computer tools.

\section{References}

[1] J.Meng: management observation, Vol.3 (2011) p.144.

[2] Y.Y.Gao: Network and Information, Vol.8 (2011) p.55.

[3] X.H.Cheng: Guangxi Light Industry, Vol.5 (2009) p.59. 